www.jmscr.igmpublication.org

Impact Factor (SJIF): 6.379

Index Copernicus Value: 79.54

ISSN (e)-2347-176x ISSN (p) 2455-0450

crossrefDOI: https://dx.doi.org/10.18535/jmscr/v6i8.188

Journal Of Medical Science And Clinical Research

IGM Publication

An Official Publication of IGM Publication

\title{
Guillain Barre Syndrome in Pregnancy- A Rare Case Report
}

\section{Sharath Kumar D Shah ${ }^{1}$, B L Girish ${ }^{2}$, L Dwarakanath ${ }^{3}$, Sahu Samra $^{4 *}$ Kadubandi Sunil Kumar}

${ }^{1}$ Professor \& Head of Department of General Medicine, Sri Siddhartha Medical College Hospital \& Research Centre, Tumakuru

${ }^{2}$ Associate Professor, Department of Obstetrics \& Gynecology, Sri Siddhartha Medical College Hospital \&

Research Centre, Tumakuru

${ }^{3}$ Professor, Department of Obstetrics \& Gynecology, Sri Siddhartha Medical College Hospital \& Research Centre, Tumakuru

${ }^{4}$ Post Graduate, Department of Obstetrics \& Gynecology, Sri Siddhartha Medical College Hospital \& Research Centre, Tumakuru

${ }^{5}$ Post Graduate, Department of General Medicine, Sri Siddhartha Medical College Hospital \& Research Centre, Tumakuru

*Corresponding Author

Sahu Samra

Email: samrasahu@gmail.com, Mob: 9945117641

\begin{abstract}
In pregnancy, Guillain Barre syndrome $(G B S)$ is rare with an estimated incidence of 1.2-1.9 per one lakh annually ${ }^{I}$. It is the most common cause of acute flaccid paralysis affecting all age groups ${ }^{2}$. It can be associated with high maternal and perinatal morbidity if it is not identified and treated promptly. A high index of suspicion, supportive measures, access to intensive care unit and Intravenous immunoglobulin/ Plasmapheresis/ Steroids therapy are cornerstones of management in GBS complicating pregnancy. Surgery and anaesthesia maybe triggers for relapse in association with an overall increase in pro inflammatory cytokines in post partum period. Neurologists and Obstetricians should be aware of the risks of relapsing GBS in the immediate post partum period.

We are reporting a rare case of GBS complicating pregnancy in $2^{\text {nd }}$ trimester. She made a good recovery with steroids and supportive measures.

Keywords: Guillain Barre syndrome, polyradiculoneuropathy.
\end{abstract}

\section{Introduction}

Guillain Barre Syndrome is an acute, frequently severe and fulminant polyradiculoneuropathy that is autoimmune in nature. A feature common to all variants of GBS is a rapidly evolving polyradiculoneuropathy preceded by a triggering event, most often an infection. It manifests as a symmetric motor paralysis, areflexia, with or without sensory, autonomic disturbances and albumin cytological dissociation. The pathogenesis of GB syndrome is unknown, but it thought to be due to molecular mimicry between 
epitopes found on cell walls of micro organisms and gangliosides found on Schwann cell membrane ${ }^{2}$. Delayed diagnosis is common in pregnancy or immediate post partum period because the initial non specific symptoms may mimic physiological changes in pregnancy ${ }^{3}$. GBS can be considered in any pregnant patient complaining of muscle weakness, general malaise, tingling of fingers and respiratory difficulty ${ }^{4,5}$. Relapse has been reported to occur in $5.5-6.8 \%$ of patients ${ }^{6}$.

\section{Case report}

This is a case report of a 35 year old female who presented to Department of Medicine at 24 weeks of gestation in her first pregnancy with Complains of weakness of both lower limbs since 1 month\& weakness of both upper limbs since 15days. There was no history suggestive of bulbar, respiratory, autonomic or cranial nerve involvement. There was history of fever $\&$ loose stools 10days prior to the onset of weakness of lower limbs which had lasted for 2days. She had received immunisation for tetanus in view of pregnancy but otherwise her progress during this pregnancy has been unremarkable. Neurological examination revealed reduced tone, power in both lower limbs. Deep tendon reflexes were absent in both lower limbs, bilaterally plantar reflexes were mute. Sensory system was involved in lower limbs (touch, temperature, vibration). Respiratory examination was within normal limits.

Serum chemistry, hemogram \& urine analysis were normal. No atypical cells were seen in peripheral smear. Serological tests for HIV, HbSAg, HCV, VDRL were negative. Thyroid function tests were within normal limits. Toxoplasma, Rubella, Cytomegalovirus \&herpes simplex virus antibodies, CSF analysis were not done due to lack of consent. Nerve Conduction Study was done which showed severe Sensori motor axonal polyradiculoneuropathy.

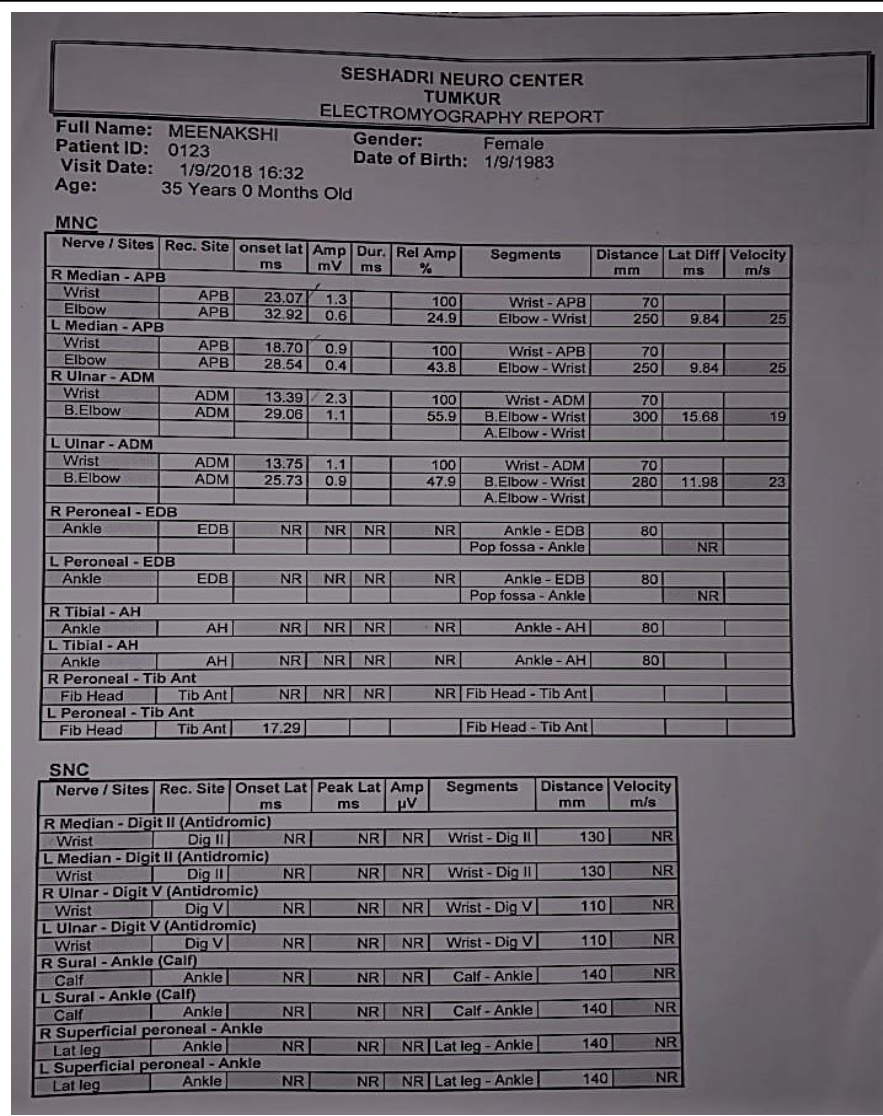

MRI Cervical spine was done which ruled out compression myelopathy.

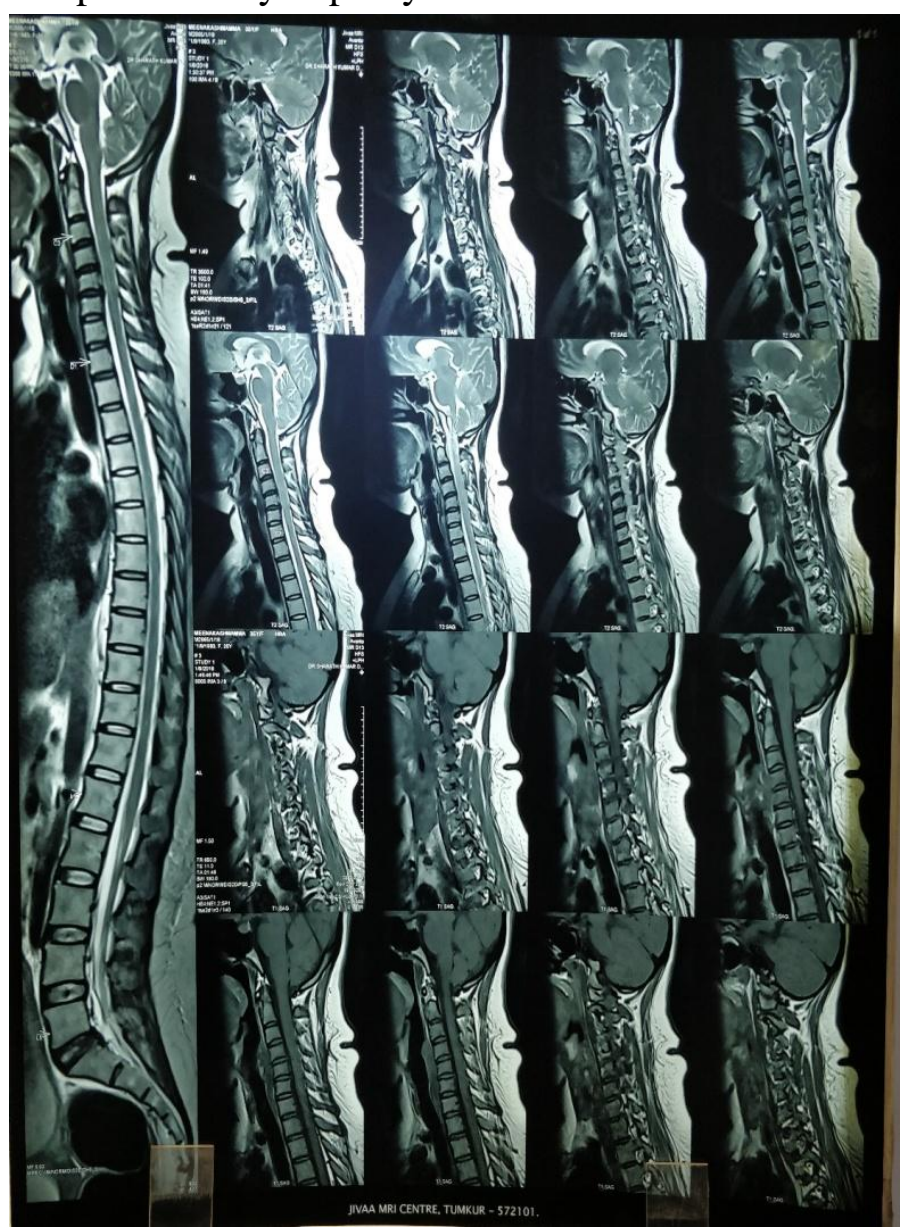

Weakness did not evolve. 
A diagnosis of Guillain Barre syndrome was made $\&$ plan of management included Plasmapheresis, IVIg or Steroids. The patients attenders did not consent for the use of Plasmapheresis or intravenous immunoglobulin. After a Glucose challenge test \& Anomaly scan which was normal, she was started on Tab. Methyl Prednisone (Category C drug) 1 gram for 3 day, Tab. Methyl Prednisone 40mg tapered and stopped over next 7 days. She was undergoing physiotherapy.

She started improving and over next 3 months she was able to walk with support \& upper limb power had improved to normal power. She could walk unsupported though with mild unsteadiness, when she was discharged. She remained neurologically stable subsequently \& was admitted in her $37^{\text {th }}$ week of gestation with high blood pressure recording. PIH profile was within normal limit. Antepartum fetal surveillance was non reassuring. She was taken up for Caesarean section under spinal anaesthesia. Indication was precious pregnancy with fetal distress.

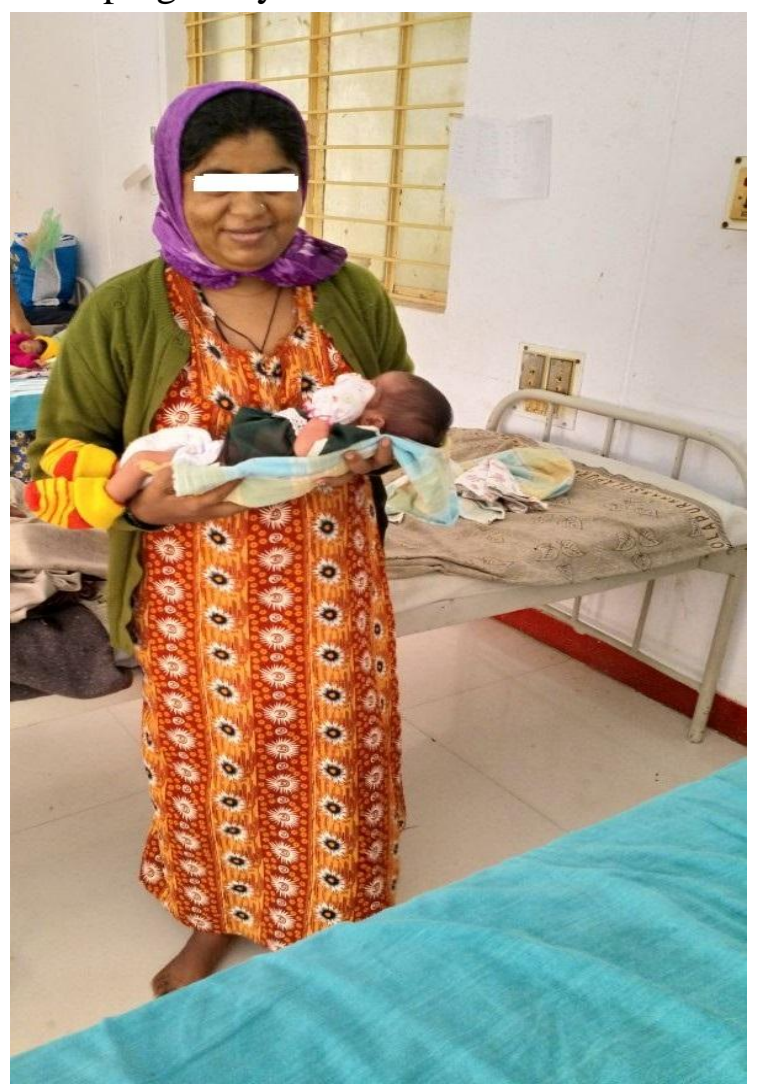

Intra op period was uneventful. Post partum period was uneventful \& she was discharged on post op day 9.She remained neurologically asymptomatic subsequently.

\section{Discussion}

GBS is a rare occurrence in pregnancy but can be associated with severe co morbidities if unrecognised, especially respiratory muscle involvement and dysautonomia. GBS is an immune mediated neurological disorder resulting primarily in muscle paralysis, which in most cases is symmetrical. About two thirds of patients have an infection within the previous 4-6weeks, most commonly a flu like illness or gastroenteritis. Implicated infectious agents include Mycoplasma pneumonia, Campylobacter jejuni, Cytomegalovirus, Epstein Barr virus.

Pregnancy is associated with a decrease in cellular immunity and increase in humoral immunity; this shift is because of production of IL10. Obstetricians should have a high index of suspicion if a pregnant woman complains of muscle weakness or breathlessness in the context of recent diarrhoeal illness or a viral infection. Diagnosis is usually made on clinical grounds supported by CSF examination, serology and nerve conduction studies. Postpartum relapses can occur. The efficacy of IVIg is well established and can be safely given during pregnancy. Judicious use of steroids is also beneficial as seen in this case.

After pregnancy is terminated, this is reversed with an overall increase in pro inflammatory cytokines ${ }^{7}$ and this accounts for the increased incidence and worsening of symptoms in the post partum period particularly during the first 2 weeks post partum ${ }^{8,9}$. Up to $20 \%$ of patients are disabled after 1 year and a maternal mortality of $7 \%$ has been quoted (non pregnant GBS has mortality $<5 \%)^{10}$. The management of GBS in pregnancy is similar to that in the non pregnant population and includes Intravenous Immunoglobulin, Plasmapheresis, ventilator support wherever required and other supportive measures like identification and treatment of infections, prophylaxis for venous thromboembolism, pain management and management of psychosocial distress resulting from the disease. GBS occurring in pregnancy is associated with an increased need 
for ventilator support and an increase in maternal mortality. In cases requiring ventilator support, the risk of premature birth has been noted to be greatly increased. Immunomodulation with IVIg or plasmapheresis has been found to improve treatment outcomes with full recovery in $70-80 \%$ of patients.

Most of the patients can be left for spontaneous labour; abortion and Caesarean section is not considered to be indicated.

Poor prognosis in GBS has been associated with rapid onset of illness, severe degree of paralysis, muscle wasting, prolonged period of peak paralysis lasting more than 2 weeks and a delay in onset of recovery lasting more than 3 weeks as well as respiratory involvement.

\section{Conclusion}

Early diagnosis, multidisciplinary input and prompt immune modulatory therapy are the cornerstones in management of GBS during pregnancy and post partum to improve outcomes for the mother and the fetus.

\section{References}

1. Visser LH, van der Meche FG, Meulstee J. Cytomegalovirus infection and Guillain Barre syndrome: the clinical, electrophysiologic and prognostic features. Dutch Guillain Barre study group. Neurology. 1996;47(3):668-73.

2. Matsuzawa Y, Sakakibara R, Shoda T. Good maternal and foetal outcomes of predominantly sensory Guillain Barre syndrome in pregnancy after intravenous immunoglobulin. Neurol Sci. 2010;31 (2):201-3.

3. Zafar MS, Naqash MM, Bhat TA, Malik GM. Guillain Barre syndrome in pregnancy: An unusual case. J Family Med Prim Care 2013;2:90-1.

4. Hughes RA, Cornblath DR. Guillain Barre syndrome. Lancet. 2005;366:1653-66. [PubMed]
5. Vijayaraghavan J, Vasudevan D, Sadique N, Rajeswari KS, Pondurangi M, Jayshree. A rare case of Guillain Barre syndrome with pregnancy. J Indian Med Assoc. 2006;104:269-70. [PubMed]

6. Taly AB, Gupta SK, Anisya V, Shankar SK, Rao S, Ras KB, et al. Recurrent Guillain Barre syndrome: A clinical, electrophysiological and morphological study. J Assoc Physicians India 1995;43:249-52.

7. Lee LK. Physiological adaptations of pregnancy affecting the nervous system. Semin Neurol. 2007;27:405-10. [PubMed]

8. Brooks H, Christian AS, May AE. Pregnancy, anaesthesia and Guillain Barre syndrome. Anaesthesia 2000;55:894-8.

9. Cheng Q, Jiang GX, Fredrikson S, Link H, de Pedro-Cuesta J. Increased incidence of Guillain Barre syndrome postpartum. Epidemiology. 1998;9:601-4.

10. Furara S, Maw M, Khan F, Powell K. Weakness in pregnancy- expect the unexpected. Obstetric Medicine. 2008;1:99-101. 